\title{
Enhancement of the photoluminescence intensity of a single InAs/GaAs quantum dot by separate generation of electrons and holes
}

\author{
(C) V. Donchev ${ }^{*, * *}$, E.S. Moskalenko,,***, K.F. Karlsson*, P.O. Holtz* , B. Monemar*, \\ W.V. Schoenfeld ${ }^{* * * *}$, J.M. Garcia***** , P.M. Petroff**** \\ * Department of Physics and Measurement Technology, Linköping University, \\ S-58183 Linköping, Sweden \\ ** Faculty of Physics, Sofia University, \\ 1164 Sofia, Bulgaria \\ *** A.F. loffe Physical-Technical Institute, Russian Academy of Sciences, \\ 194021 St. Petersburg, Russia \\ **** Materials Department, University of California, \\ Santa Barbara, California 93106 USA \\ ***** Instituto de Microelectronica de Madrid, CNM-CSIC Isaak Newton 8, PTM, \\ 28760 Tres Cantos, Madrid, Spain \\ E-mail: evgenii.moskalenko@mail.ioffe.ru
}

(Received January 24, 2006)

It is demonstrated that the micro-photoluminescence $(\mu \mathrm{PL})$ spectrum of a single InAs/GaAs self-assembled quantum dot (QD) undergoes considerable changes when the primary laser excitation is complemented with an additional infrared laser. The primary laser, tuned slightly below the GaAs band gap, provides electron-hole pairs in the wetting layer (WL), as well as excess free electrons from ionized shallow acceptors in the GaAs barriers. An additional IR laser with a fixed energy, well below the QD ground state transition, generates excess free holes from deep levels in GaAs. The excess electron and hole will separately experience a diffusion, due to the time separation between the two events of their generation, to eventually become captured into the QD. Although the generation rates of excess carries are much lower than that of the electron-hole pairs generation in the WL, they influence considerably the QD emission at low temperatures. The integrated PL intensity increases by several times compared to single laser excitation and the QD exciton spectrum is redistributed in favor of a more neutral charge configuration. The dependence of the observed phenomenon on the powers of the two lasers and the temperature has been studied and is in consistence with the model proposed. The concept of dual excitation could be successfully applied to different low-dimensional semiconductor structures in order to manipulate their charge state and emission intensity.

This work was supported by grants from the Swedish Foundation for Strategic Research (SSR) and Swedish Research Council (VR). Financial support from the Wenner-Gren Foundation and the program „Low-Dimensional Quantum Structures“ of the Russian Academy of Sciences is achnowledged by E.S.M. V.D. is thankful for financial support from the Swedish Foundation for International Cooperation in Research and Higher Education (STINT) and from the Bulgarian National Science Fund.

PACS: 78.67.Hc, 71.55.Eq, 73.50.Gr

\section{Introduction}

In photoluminescence (PL) studies of low-dimensional semiconductor structures, the optical generation of free carriers normally takes place in the barriers, while the desired radiative recombination, at least at low temperatures, occurs after carrier diffusion into the lowest energy state of the potential well. In the commonly employed single laser excited PL, the electron $(e)$ and the hole $(h)$ in the $e-h$ pair are generated simultaneously by the same photon and experience a joint diffusion, which results in a considerable emission in the barriers with rather short recombination times $(\leq \mathrm{ns}[1,2])$. However, if the electrons and the holes are generated not as a pair, but separately in time (and space), they will diffuse separately, to be captured and recombine in the potential minimum, resulting in an enhanced PL emission. The recombination in the barriers can be avoided, if the time interval between the two events of generation of the electron and the hole is large compared to the time for carrier migration, capture and relaxation to the lowest energy state (typically some tens of ps [3]). Such separate generation of electrons and holes can be realized e. g. by means of two excitation sources initiating different electronic transitions involving defect levels.

Semiconductor quantum dots (QDs) effectively confine electrons and holes on the nanometer length scale in all three directions and are a typical example of potential wells surrounded by barriers, where the optical generation of free carriers normally takes place. Recent modeling of QD lasers [4] has emphasized the importance of the carrier capture mechanism on the threshold current, with a resulting larger current for exciton capture than for separate electron and hole capture. Mazurenko et al. [5] reported on an infrared (IR) laser induced increase (up to 40\%) of the PL output from InAs/GaAs QD ensembles, excited with photon energy above the GaAs band gap. This 
observation was explained in terms of an optical release of carriers previously captured from the QDs into deep traps [5]. However, due to the high dot density used [5], the authors were neither able to observe any PL signal from the wetting layer (WL), nor to distinguish between different charged exciton complexes in the QD PL. An enhancement of the radiative efficiency due to photoionization of deep levels (DLs) by an additional below-band-gap excitation was observed also in epitaxial GaAs [6].

The spectroscopy of a single QD has proven to be an extremely sensitive probe to excess charges in the QD and its surrounding [7-12], since it allows monitoring of the spectral fine structure related to different charged exciton complexes. In our previous studies [9-12] on InAs/GaAs QD structures with a dot density as low as $\sim 10^{6} \mathrm{~cm}^{-2}$ (allowing simultaneous monitoring of the PL emission from a single QD and the surrounding WL), we have demonstrated that a proper selection of the photon excitation energy $h v_{\mathrm{ex}}$, will allow single charge carriers, electrons and/or holes, to be generated in the sample and subsequently be captured by the QDs. This is reflected in the charge distribution of the QD excitonic states, as monitored by low-temperature $(5 \mathrm{~K})$ micro-PL $(\mu \mathrm{PL})$ spectra of the individual QD.

In this paper, we report on dual laser excited $\mu \mathrm{PL}$ studies of self-assembled InAs/GaAs single QDs. The primary laser, tuned in the range $E_{\mathrm{th}}<h v_{\mathrm{ex}}<E_{X}^{\mathrm{GaAs}}$, provides $e-h$ pairs via photon absorption in the WL, as well as excess free electrons from shallow acceptors in the GaAs barriers [11]. An additional IR laser generates excess holes via electronic excitations involving DLs. The powers of the two lasers are chosen in such a way, that the average time interval between two individual events of an excess electron and an excess hole generation is much longer than the time required for carrier migration in the WL and collection into the QDs. When the principal excitation is complemented with the IR laser, it is found that the QD PL intensity increases several times, while the WL PL remains almost unchanged. Another striking observation is the charge redistribution monitored in the QD $\mu \mathrm{PL}$ spectrum. With dual laser excitation, the QD becomes more neutral. The dependence of these interesting effects on the laser powers and the temperature is studied.

\section{Samples and experimental set-up}

The sample studied was grown by molecular-beamepitaxy (MBE) employing a special growth procedure to obtain a very low QD density, corresponding to an average inter-dot spacing of about $10 \mu \mathrm{m}$. The self-assembled InAs QDs are lens-shaped with a typical lateral size (height) of $35 \mathrm{~nm}(4.5 \mathrm{~nm})$. They are located on a thin $(\sim 0.5 \mathrm{~nm})$ WL sandwiched between two $100 \mathrm{~nm}$ thick layers of GaAs. A short-period $40 \times 2 \mathrm{~nm} / 2 \mathrm{~nm}$ AlAs/GaAs superlattice is used as a buffer layer between the structure and the semiinsulating GaAs substrate.
A conventional diffraction-limited $\mu \mathrm{PL}$ setup was used in these studies. The excitation of a single QD was performed by two cw Ti- $S p$ laser beams, focused on the sample surface down to a $2 \mu \mathrm{m}$ spot by means of a microscope objective. The primary laser, used to excite the PL, was tuned from $h v_{\mathrm{ex}}=1.493$ to $1.515 \mathrm{eV}$, while the second laser was set to a fixed energy in IR, $h v_{\mathrm{IR}}=1.233 \mathrm{eV}$. The power of the primary laser $P_{0}$ was varied in the range $17 \mathrm{nW}<P_{0}<10 \mu \mathrm{W}$, while the IR laser power was varied in the range $0.55<P_{\mathrm{IR}}<100 \mu \mathrm{W}$. The PL signal was dispersed by a single grating $0.45 \mathrm{~m}$ monochromator and detected by a liquid-nitrogen-cooled Si CCD camera. The measurements were performed at temperatures $T$ in the range $5<T<65 \mathrm{~K}$ using a continuous flow cryostat. Eight single QDs were studied, revealing a similar behavior as the QD presented here. A detailed description of the sample and the experimental set-up is given elsewhere [9-11].

\section{Experimental results and discussion}

The threshold energy $E_{\mathrm{th}}$ described above determines the primary laser excitation energy above which excess electrons are generated. This threshold energy is defined by $E_{\text {th }}=E_{g}^{\mathrm{GaAs}}-E_{A}$, where $E_{g}^{\mathrm{GaAs}}=1.519 \mathrm{eV}$ is the GaAs band gap and $E_{A}$ is the shallow acceptor ionization energy. The values of $E_{\text {th }}$ have been found to be either 1.483 or $1.493 \mathrm{eV}$ [11] (depending on the QD surrounding) in accordance with the ionization energies of the main residual acceptors in MBE grown GaAs: $\mathrm{Si}\left(E_{A}=34 \mathrm{meV}\right)$ and $\mathrm{C}$ $\left(E_{A}=26 \mathrm{meV}\right)$, respectively [13]. The results presented in this paper are for $h v_{\mathrm{ex}} \geq 1.493 \mathrm{eV}$, which implies that electrons from both types of acceptors are excited in the GaAs conduction band by the photons of the primary laser.

The solid curves in Fig. 1 show $\mu$ PL spectra of the QD measured at $(a) 5$ and $(b) 55 \mathrm{~K}$ under single laser excitation with $h v_{\mathrm{ex}}=1.503 \mathrm{eV}$. The dotted curves represent $\mu \mathrm{PL}$ spectra recorded under the same experimental conditions, but with an additional IR laser excitation. Altogether three emission lines, marked as $X, X^{-}$and $X^{2-}$, can be seen on Fig. 1. They have been identified as the neutral $(X)$, single $\left(X^{-}\right)$and double $\left(X^{2-}\right)$ negatively charged exciton [10], corresponding to the $1 e 1 h, 2 e 1 h$ and $3 e 1 h$ QD ground state charge configurations, respectively. The small peak labeled $X^{3-}$ is tentatively ascribed to the triple negatively charged exciton $(4 e 1 h)$.

The $\mu \mathrm{PL}$ spectrum, obtained at $5 \mathrm{~K}$, excited with a single laser is dominated by the doubly negativety charged exciton $X^{2-}$. This is observed to be dominant for all $h v_{\text {ex }}$ in the range $E_{\mathrm{th}}<h v_{\mathrm{ex}}<E_{X}^{\mathrm{GaAs}}$. The predominance of $X^{2-}$ is explained in terms of excess electrons generated via optical electronic transitions from shallow acceptors to the conduction band in the GaAs barriers [11] (represented by arrow 1 in Fig. 2). Under dual laser excitation, the $\mu \mathrm{PL}$ spectrum changes dramatically: the line $X^{2-}$ is quenched and, instead, the neutral exciton $X$ becomes predominant. Besides, an overall increase of the integrated QD PL 


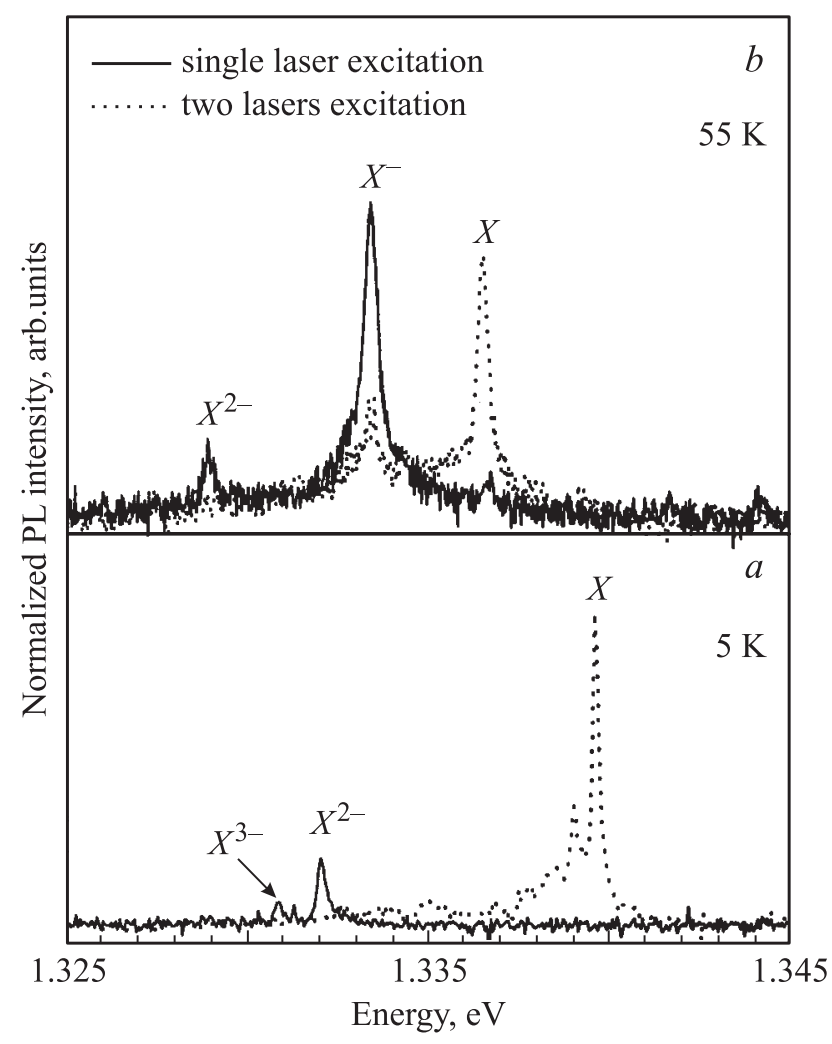

Figure 1. Micro-PL spectra of a single InAs/GaAs QD under single (solid lines) and dual (dotted lines) laser excitation measured at $(a) 5$ and $(b) 55 \mathrm{~K}$ with $h v_{\mathrm{ex}}=1.503 \mathrm{eV}, P_{0}=40 \mathrm{nW}$, and $P_{\mathrm{IR}}=100 \mathrm{muW}$.

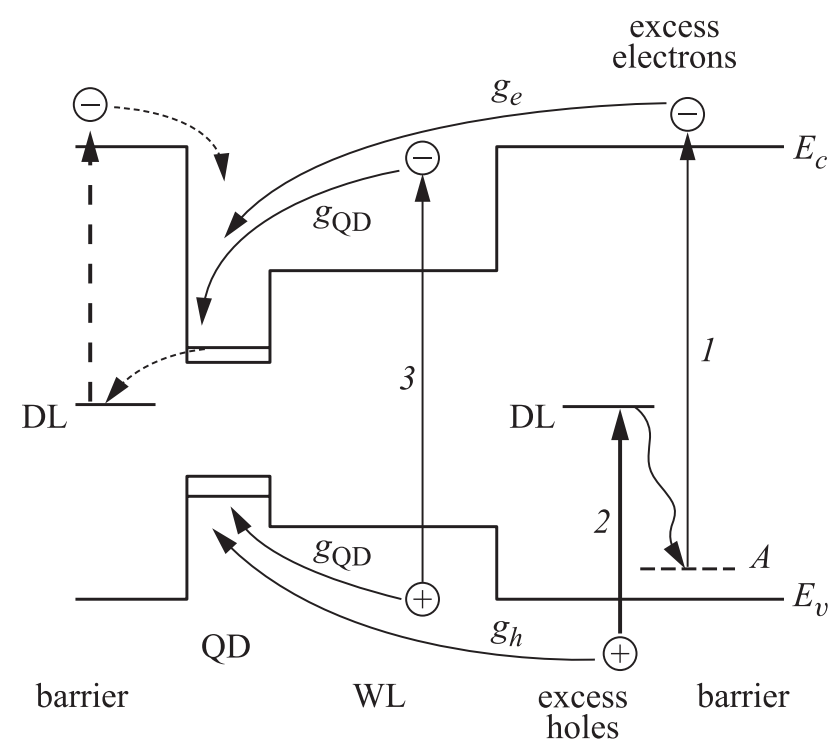

Figure 2. A schematic reqresentation of the conduction band $\left(E_{c}\right)$ and valence band $\left(E_{v}\right)$ edges in the growth direction together with the acceptor $(A)$ and the deep level (DL) positions in the sample studied. The thin (thick) vertical arrows correspond to the primary (IR) laser excitation, while the curved arrows represent the capture processes into the QD and the deep levels discussed in the text. The undulated arrow represents the electron capture from the deep levels to the shallow acceptors. intensity $I_{\mathrm{PL}}^{\mathrm{QD}}$ by more than 5 times relatively to the single laser excited PL intensity is observed. For all $h v_{\text {ex }}$ in the above-mentioned range, the addition of the IR laser i) redistributes the $\mu \mathrm{PL}$ spectrum towards a more neutral state, i. e. in favor of the $X$ PL line and ii) increases $I_{\mathrm{PL}}^{\mathrm{QD}}$ several times. The IR laser alone does not give any detectable PL, as expected from its energy, which is well below the QD emission energy.

No change which could be induced by the IR laser is detected for the dot PL intensity, when the QD is excited directly, i.e. for $h v_{\mathrm{ex}}$ below the absorption edge of the WL. This allows us to exclude capture processes of carriers from the QD to DLs followed by an optical excitation to the barriers and recapture into the dot (see dashed arrows in Fig. 2), as has been considered earlier [5]. Further, it is important to note that the considerable increase of $I_{\mathrm{PL}}^{\mathrm{QD}}$ ( $>5$ times) is not accompanied by an analogous increase of the integrated $\mu \mathrm{PL}$ intensity of the $\mathrm{WL}, I_{\mathrm{PL}}^{\mathrm{WL}}$, which increases only by $5-10 \%$. Taking into account all the experimental observations described above, the IR laser induced changes in the PL spectra can be understood in terms of generation of excess holes. Since $h v_{\mathrm{IR}}$ is in the extrinsic region of the sample, we assume that the holes are generated by optical electronic transitions between the valence band and DLs in the GaAs (represented by arrow 2 in Fig. 2). As the QD is already populated with excess electrons by the primary laser (see above), excess positive charges are effectively attracted. This increases the probability for a more neutral charge distribution, i.e. for the $2 e 1 h$ and $1 e 1 h$ charge configurations $\left(X^{-}\right.$and $\left.X\right)$. The observed increase of $I_{\mathrm{PL}}^{\mathrm{QD}}$ can be explained by the combined generation of excess electrons by the primary laser and excess holes by the IR laser with generation rates $g_{e}$ and $g_{h}$, respectively. A permanent generation of excess electrons and holes requires a continuous replenishment of the acceptors with electrons and the DLs with holes. Most likely this occurs by electron capture from the DLs to the acceptors (represented by undulated arrow in Fig. 2). It is worth noting that PL measurements of GaAs have suggested that the acceptors tend to cluster in the neighborhood of the DL centers [14].

The generation rate of additional $e-h$ pairs in the QD $g_{\text {ad }}$ is on top of the generation rate $g_{\mathrm{QD}}$, originating from the primary laser absorption in the WL (represented by arrow 3 in Fig. 2). $g_{\mathrm{QD}}$ is in fact the observed $I_{\mathrm{PL}}^{\mathrm{QD}}$ without the IR laser excitation. $I_{\mathrm{PL}}^{\mathrm{WL}}$ could be considered to be equal to the carrier generation rate in the $\mathrm{WL}$ $g_{\mathrm{WL}}$. Introducing a collection efficiency $w_{c}$ of the photo carriers from the $\mathrm{WL}$ to the $\mathrm{QD}$ as $w_{c}=I_{\mathrm{PL}}^{\mathrm{QD}} / I_{\mathrm{PL}}^{\mathrm{WL}}, g_{\mathrm{QD}}$ can be expressed as $g_{\mathrm{QD}}=w_{c} g_{\mathrm{WL}}=w_{c} \alpha_{\mathrm{WL}} d_{\mathrm{WL}} P_{0} / h v_{\mathrm{ex}}$, where $d_{\mathrm{WL}}=0.5 \mathrm{~nm}$ is the WL thickness and in a first approximation the absorpsion coefficient of bulk InAs at $h v_{\mathrm{ex}}, \alpha_{\mathrm{WL}}=10^{4} \mathrm{~cm}^{-1}[15]$ is used. From the expression $w_{c}=I_{\mathrm{PL}}^{\mathrm{QD}} / I_{\mathrm{PL}}^{\mathrm{WL}}, w_{c}$ can be evaluated to be $\approx 2 \cdot 10^{-3}$ for $h v_{\mathrm{ex}}$ used in this study. 
Both $g_{e}$ and $g_{h}$ are linearly proportional to the excitation power of the corresponding laser, $P_{0}$ and $P_{\mathrm{IR}}$, respectively, as given by: $g_{e}=\sigma_{e} N_{a} d_{\mathrm{GaAs}} P_{0} / h v_{\mathrm{ex}}$ and $g_{h}=\sigma_{h} N_{\mathrm{DL}} d_{\mathrm{GaAs}} P_{\mathrm{IR}} / h v_{\mathrm{IR}}$. Here $d_{\mathrm{GaAs}}=200 \mathrm{~nm}$ is the thickness of the GaAs barriers, $\sigma_{e}\left(\sigma_{h}\right)$ is the acceptor (DL) optical cross section for electrons (holes) and $N_{a}\left(N_{\mathrm{DL}}\right)$ is the concentration of ionized acceptors (empty DLs) in the GaAs. To get an assessment of $g_{e}$ and $g_{h}$, typical employed values are: $N_{\mathrm{DL}}=5 \cdot 10^{12} \mathrm{~cm}^{-3}$ [16], $N_{a}=6 \cdot 10^{13} \mathrm{~cm}^{-3} \quad[17], \quad \sigma_{h}=6 \cdot 10^{-18} \mathrm{~cm}^{2}$ (the value for the EL2 DL in GaAs at $1.233 \mathrm{eV}$ [18]) and $\sigma_{e}=3.5 \cdot 10^{-15} \mathrm{~cm}^{2}$ for the carbon acceptor. ${ }^{1}$ Thus, for the lowest value of $P_{0}(17 \mathrm{nW})$ and the highest value of $P_{\mathrm{IR}}(100 \mu \mathrm{W})$, we obtain $g_{e} \approx g_{h} \approx 3 \cdot 10^{5} \mathrm{~s}^{-1}$. Since $g_{\text {ad }}$ is determined by the lower value of $g_{e}$ or $g_{h}$, one can consider $g_{\text {ad }}=g_{h}$ for all laser powers used in this study.

The increase of $I_{\mathrm{PL}}^{\mathrm{WL}}$ is relatively small due to the fact that the excess electrons and holes are generated by two different photons, i.e., in two different moments separated by a time interval of the order of the lower value of $g_{e}^{-1}$ or $g_{h}^{-1}$. This interval is at shortest $5.7 \mathrm{~ns}$ for the highest $P_{0}$ used here $(10 \mu \mathrm{W})$, i. e., much longer than the time needed by the photo-excited carrier for migration, capture and relaxation to the lowest QD energy level ( $<1 \mathrm{~ns}[3])$. Consequently, the excess electron and hole will likely be captured and recombine in the QD rather than in the WL. Accordingly, in the expressions for $g_{e}$ and $g_{h}$ of our simplified estimate, it is assumed that all excess carriers are efficiently collected into the QD, as earlier demonstrated for excess electrons [11].

In order to understand the increase of $I_{\mathrm{PL}}^{\mathrm{QD}}$, the rate $R_{\mathrm{QD}}$ is introduced, defined as the ratio between $I_{\mathrm{PL}}^{\mathrm{QD}}$ measured under dual and single laser excitation, respectively, i.e., $R_{\mathrm{QD}}=I_{\mathrm{PL}}^{\mathrm{QD}}\left(P_{\mathrm{IR}}\right) / I_{\mathrm{PL}}^{\mathrm{QD}}\left(P_{\mathrm{IR}}=0\right)$. According to the abovedescribed model, $R_{\mathrm{QD}}$ should be expressed as

$$
R_{\mathrm{QD}}=\frac{g_{\mathrm{QD}}+g_{\mathrm{ad}}}{g_{\mathrm{QD}}}=1+\frac{g_{\mathrm{ad}}}{g_{\mathrm{QD}}} .
$$

The dependence of $R_{\mathrm{QD}}$ on the powers ratio of the two lasers, $P_{\mathrm{IR}} / P_{0}$, wirh either $P_{0}$ or $P_{\mathrm{IR}}$ fixed is shown in Fig. 3. For high $P_{0}$ 's and/or low $P_{\mathrm{IR}}$ 's, $R_{\mathrm{QD}}$ is close to unity, since $g_{\text {ad }} / g_{\text {QD }} \ll 1$ (cf. Eq. (1)). This fact is in agreement with the experimental observation that no redistribution of the $\mu \mathrm{PL}$ spectrum occurs (not shown here). $R_{\mathrm{QD}}$ increases for low $P_{0}$ 's and/or high $P_{\mathrm{IR}}$ 's, because $g_{\text {ad }} / g_{\mathrm{QD}}>1$ in this case (cf. Eq. (1)). The experimental points are well fitted by the linear expression $R_{\mathrm{QD}}=1+a\left(P_{\mathrm{IR}} / P_{0}\right)$ with $a=1.1 \cdot 10^{-3}$ (Fig. 3). According to Eq. (1) and the expressions for $g_{h}$ and $g_{\mathrm{QD}}$ with $w_{c}\left(h v_{\mathrm{ex}}=1.503 \mathrm{eV}\right)=1.7 \cdot 10^{-3}, a$ should be $\sigma_{h} N_{\mathrm{DL}} d_{\mathrm{GaAs}} h v_{\mathrm{ex}} /\left(w_{c} \alpha_{\mathrm{WL}} d_{\mathrm{WL}} h v_{\mathrm{IR}}\right)=0.9 \cdot 10^{-3}$. This is in good correspondence with the experimental value, which further supports the proposed model.

In a complementary experiment, the spot of the primary laser (with $h v_{\mathrm{ex}}=1.508 \mathrm{eV}$ ) was moved aside of the QD

\footnotetext{
${ }^{1}$ Calculated for $h v_{\mathrm{ex}}=1.503 \mathrm{eV}$ by means of the formulae given in W.P. Dumke, Phys. Rev. 132 (5), 1998 (1963).
}

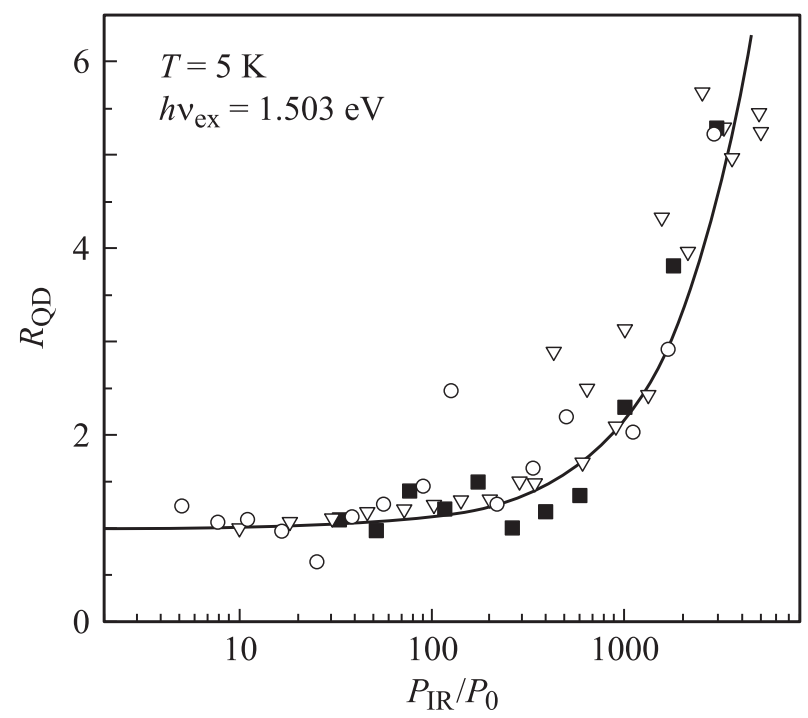

Figure 3. The symbols show the ratio $R_{\mathrm{QD}}$ of the spectrally integrated QD PL intensities measured under dual and single laser excitation, respectively, at $T=5 \mathrm{~K}$ with $h v_{\mathrm{ex}}=1.503 \mathrm{eV}$ as a function of the power ratio of the two lasers, $P_{\mathrm{IR}} / P_{0}$, as follows: circles $-P_{\mathrm{IR}}=50 \mu \mathrm{W}$ and $P_{0}$ in the range 17 to $10000 \mathrm{nW}$; triangles $-P_{\mathrm{IR}}=100 \mu \mathrm{W}$ and $P_{0}$ in the range 17 to $10000 \mathrm{nW}$; and squares $-P_{0}=17 \mathrm{nW}$ and $P_{\mathrm{IR}}$ in the range 0.55 to $50 \mu \mathrm{W}$. The solid line is calculated, based on the expression $R_{\mathrm{QD}}=1+a\left(P_{\mathrm{IR}} / P_{0}\right)$ with $a=1.1 \cdot 10^{10^{-3}}$.

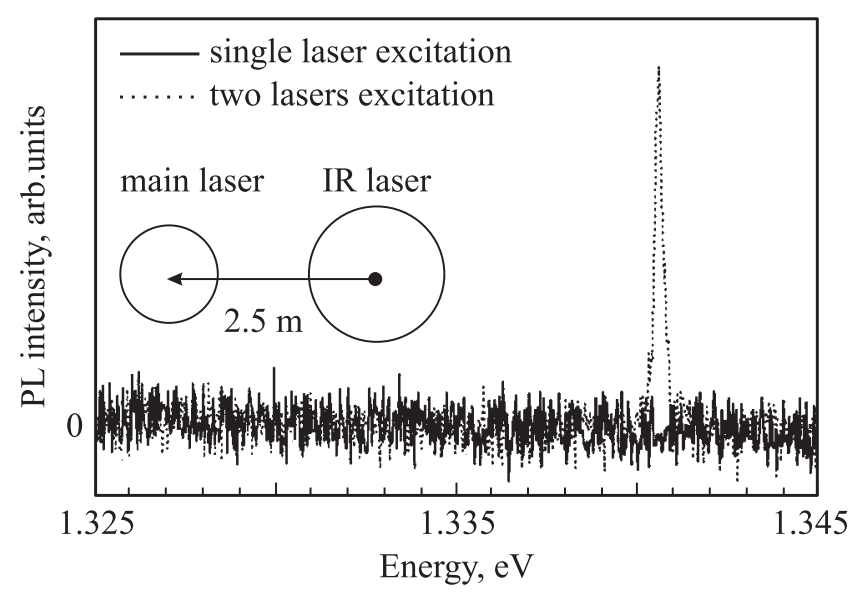

Figure 4. Micro-PL spectra of a single InAs/GaAs QD under single (solid line) and dual (dotted line) laser excitation measured at $5 \mathrm{~K}$ with $h v_{\mathrm{ex}}=1.508 \mathrm{eV}, P_{0}=20 \mathrm{nW}$, and $P_{\mathrm{IR}}=50 \mu \mathrm{W}$. The spot of the IR laser is positioned on the QD, while the spot of the primary laser is moved $2.5 \mu \mathrm{m}$ aside of the QD, as schematically shown in the inset.

by a distance of $2.5 \mu \mathrm{m}$. This distance and $P_{0}$ were chosen in such a way that the PL signal rendered undetectable under single laser excitation (see Fig. 4). The $e-h$ pairs generated in this case can not reach the QD because of the long distance, but will rather recombine in the WL. However the excess electrons are efficiently collected into the QD [11]. Indeed, when the IR laser, focussed on the 


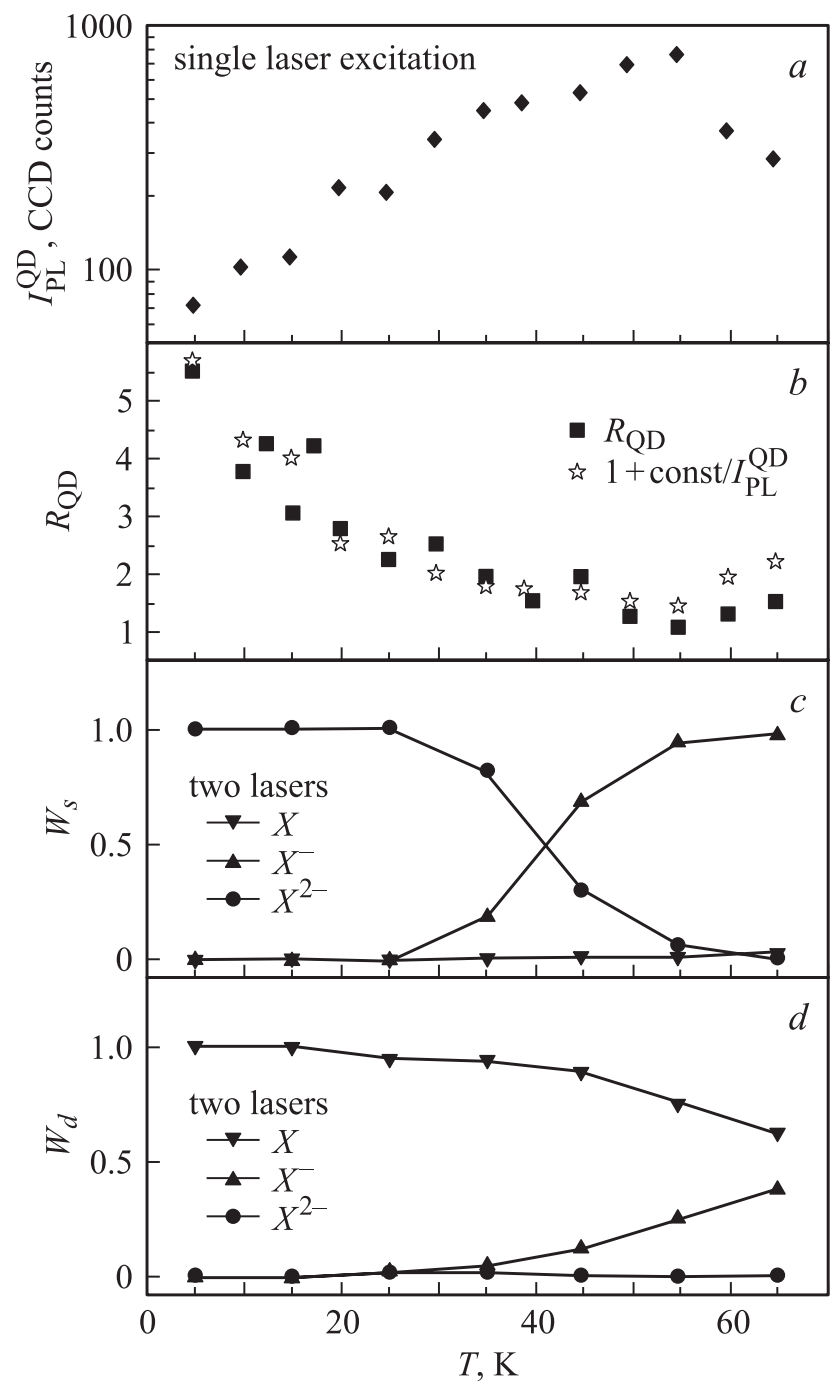

Figure 5. The temperature evolution of $(a)$ the intergrated QD $\mathrm{PL}$ intensity, $I_{\mathrm{PL}}^{\mathrm{QD}}$, measured under single laser excitation; $(b)$ the ratio $R_{\mathrm{QD}}$ of the integrated QD PL intensities measured under dual and single laser excitation, respectively and compared with the calculated values (given by stars); $(c)$ and $(d)$ the relative contributions of the neutral (done triangles), single (uptriangles) and double (circles) charged excitons to the total QD PL intensity in the case of $(c)$ single and $(d)$ dual laser excitation. $h v_{\mathrm{ex}}=1.503 \mathrm{eV}, P_{0}=40 \mathrm{nW}$, and $P_{\mathrm{IR}}=100 \mu \mathrm{W}$. The lines in $(c)$ and $(d)$ are only guides for the eyes.

QD, was added, a well-defined $\mu \mathrm{PL}$ spectrum consisting of the neutral exciton $X$ appeared (Fig. 4). This phenomenon can be explained with the assumption that each laser supplies the QD with just one type of carriers, electrons or holes, respectively. This experimental observation can be regarded as a direct proof of the effect of separate generation of excess electrons and holes by the primary and the IR laser, respectively.

Finally, the temperature dependence of the described effect with dual laser excited PL spectra has been studied (with $P_{0}=40 \mathrm{nW}$ and $P_{\mathrm{IR}}=100 \mu \mathrm{W}$ ). The increase of the temperature enhances the acceptor ionization processes from the valence band in GaAs. On the one hand, this should increase the number of the optically generated excess electrons via the ionized acceptors. On the other hand, the number of thermally generated holes from the acceptor levels in GaAs grows quickly for $T>\sim 30 \mathrm{~K}^{2}$ and becomes comparable to the number of optically generated excess electrons. The appearance of free holes in the valence band leads to an efficient band-to-band recombination, which decreases the number of the excess electrons and consequently results in a reduced charging of the QD (see the discussion in [11]). Indeed, with increasing temperature, the single laser excited $\mu \mathrm{PL}$ spectrum is gradually redistributed from the double charged $\left(X^{2-}\right)$ toward the single charged exciton line $\left(X^{-}\right)$and at $T=55 \mathrm{~K}$, the $X^{-}$line already dominates the spectrum, as shown in Fig. 1,b. This temperature induced redistribution of the PL spectrum under single laser excitation is illustrated in Fig. 5,c. The figure displays the relative contribution $W_{s}$ of the neutral together with the single and double charged excitons to the total QD PL intensity, calculated from the areas under the $X, X^{-}$ and $X^{2-}$ spectral lines, respectively. It is seen that the contribution of $X^{2}$ decreases with temperature, while that of $X^{-}$increases and becomes dominant at $T>40 \mathrm{~K}$.

Another effect of the increased temperature is the thermally enhanced exciton and carrier transport in the WL [19], resulting in a higher collection efficiency of excitons and photo-carriers from the WL to the QD. This effect explains the gradual increase of the single laser excited $I_{\mathrm{PL}}^{\mathrm{QD}}$ (and consequently $\left.g_{\mathrm{QD}}\right)$ in the range from 5 to $55 \mathrm{~K}$, shown in Fig. 5, $a$. At $T=55 \mathrm{~K}, I_{\mathrm{PL}}^{\mathrm{QD}}$ is about 10 times larger with respect to $T=5 \mathrm{~K}$. The decrease of $I_{\mathrm{PL}}^{\mathrm{QD}}$ for $T>55 \mathrm{~K}$ can be due to thermal activation of non-radiative recombination channels [20].

Concerning the IR laser generation of excess holes, we can assume that $g_{h}$ does not change significantly with increasing temperature. This is justified by the fact that in the temperature range under study, the thermal ionization of the DLs is negligible, because the DL ionization energy (of the order $0.5 E_{g}^{\mathrm{GaAs}}$ ) is much larger than $k T$. No increase of $g_{h}$ is expected, but rather a slight decrease as a result of electron trapping from the conduction band into the DL (which reduces the number of empty DLs). It is also assumed that for the experimental conditions $\left(P_{0}, P_{\mathrm{IR}}\right.$ and $\left.T\right)$ employed, $g_{h}$ remains below $g_{e}$, i.e., $g_{\text {ad }}=g_{h}$ (as estimated above for $T=5 \mathrm{~K}$ ). Taking these facts into account together with the temperature increase of $g_{\text {QD }}$ (Fig. 5, a) and Eq. (1), one expects a decrease of $R_{\mathrm{QD}}$ as a function of temperature. This has been experimentally confirmed, as shown in Fig. 5, $b$. It is seen that $R_{\mathrm{QD}}$ gradually decreases with temperature to become nearly 1 at $\sim 55 \mathrm{~K}$. Moreover, according to Eq. (1) and the above considerations, the dependence of $R_{\mathrm{QD}}(T)$ on the temperature should follow the expression $\left(1+\right.$ const $\left./ I_{\mathrm{PL}}^{\mathrm{QD}}(T)\right)$. The so-derived prediction of $R_{\mathrm{QD}}(T)$

\footnotetext{
${ }^{2}$ Calculated in dark using Fermi statistics and the carbon ionization energy $(26 \mathrm{meV})$ in the charge neutrality equation with an assumed total acceptor concentration of $1 \cdot 10^{14} \mathrm{~cm}^{-3}$.
} 
(employing the values of $I_{\mathrm{PL}}^{\mathrm{QD}}$ from Fig. 5, $a$ ) is represented in Fig. $5, b$ by the stars. As expected, the two sets of data points in Fig. 5, $b$ coincide very well. This finding gives further support to the proposed model. As the temperature increases, the relative contribution of the excess electrons and holes supplied into the QD becomes less important with respect to the increased number of $e-h$ pairs created by the primary laser in the WL and subsequently collected into the QD.

While $R_{\mathrm{QD}}$ qradually decreases with temperature, the second IR laser induced effect, the altered QD charge configuration in favor of a more neutral exciton state is clearly demonstrated even at $55 \mathrm{~K}$. This is illustrated in Fig. 1, $b$, where the doubly excited $\mu \mathrm{PL}$ spectrum is again dominated by the neutral exciton line $X$ (as was the case at $5 \mathrm{~K}$, Fig. 1, $a$ ). The temperature evolution of this effect of the IR laser is illustrated in Fig. 5, $d$. The figure displays the relative contributions $W_{d}$ of the neutral, single and double charged excitons to the total QD PL intensity in the case of dual laser excitation. It is seen that the contribution of $X$ is more than 3 times larger than the other two components for all temperatures in the range $5 \leq T \leq 55 \mathrm{~K}$. While the $X$ exciton is under the detection limit at any temperature with single laser excitation (see Fig. 5,c), the IR laser will change the QD charge configuration in favor of a more neutral exciton state in whole the temperature range under study. Nevertheless, a decreasing tendency for the $X$ exciton intensity is observed for $T>40 \mathrm{~K}$, where the contributions of $X$ and $X^{-}$are approaching (see Fig. 5, $d$ ). This behavior is consistent with the low values (close to 1 ) of $R_{\mathrm{QD}}$ in this range (see Fig. 5, $b$ ). As explained above, for $T>40 \mathrm{~K}$, the photo carriers generated as a result of the absorption of the primary laser in the WL are efficiently collected from the WL into the QD (see Fig. 5, a) and their relative contribution to the QD PL ( $\left.g_{\mathrm{QD}}\right)$ becomes more and more dominant with respect to the PL originating from the excess electrons and holes $\left(g_{\mathrm{ad}}\right)$.

\section{Conclusion}

By means of dual laser excitation of a single InAs/GaAs QD with an appropriate choice of the excitation energies and powers, the QD is supplied with separately generated excess electrons and holes from shallow acceptors and DLs, respectively, in the GaAs barriers. Although the generation rates of excess carriers are much lower than the $e-h$ pair generation rate of the primary laser in the $\mathrm{WL}$, the resulting effect is quite strong at $5 \mathrm{~K}$. The QD PL intensity increases several times compared to single laser excitation and the $\mu \mathrm{PL}$ spectrum is redistributed in favor of a more neutral exciton configuration. This is due to the fact that for the excitation powers employed, the generations of excess electrons and holes are separated in time, causing a separate diffusion with a resulting smaller probability for recombination in the WL before capture into the QD. At elevated temperatures, the $e-h$ pairs generated by the primary laser in the WL are more and more efficiently collected into the QD, due to the thermally enhanced transport in the WL, and consequently the contribution of excess electrons and holes becomes less important. As a result the effect of the QD PL intensity increase gradually weakens with temperature, while the redistribution effect exhibits a decreasing tendency above $40 \mathrm{~K}$, to get quenched above $\sim 55 \mathrm{~K}$.

\section{References}

[1] S. Raymond, K. Hinzer, S. Fafard, J.L. Merz. Phys. Rev. B 61, R16331 (2000).

[2] U. Jahn, R. Nötzel, J. Ringling, H.-P. Schönherr, H.T. Grahn, K.H. Ploog, E. Runge. Phys. Rev. B 60, 11038 (1999).

[3] S. Marcinkevičius, R. Leon. Appl. Phys. Lett. 76, 2406 (2000).

[4] M. Grundmann, D. Bimberg. Phys. Stat. Sol. (a) 164, 297 (1997).

[5] D.A. Mazurenko, A.V. Scherbakov, A.V. Akimov, A.J. Kent, M. Henini. Semicond. Sci. Technol. 14, 1132 (1999).

[6] A.V. Akimov, V.G. Shofman. J. Lumin. 53, 335 (1992).

[7] M. Lomascolo, A. Vergine, T.K. Johal, E. Rimaldi, A. Passarelo, R. Cingolani, S. Patanè, M. Labardi, M. Allegrini, F. Troiani, E. Molinari. Phys. Rev. B 66, 041302 (2002).

[8] J.J. Finley, A.D. Ashmore, A. Lemaitre, D.J. Mowbray, M.S. Skolnik, I.E. Itskevich, P.A. Maksym, M. Hopkinson, T. Krauss. Phys. Rev. B 63, 073307 (2001).

[9] E.S. Moskalenko, V. Donchev, K.F. Karlsson, P.O. Holtz, B. Monemar, W.V. Schoenfeld, J.M. Garcia, P.M. Petroff. Phys. Rev. B 68, 155317 (2003).

[10] E.S. Moskalenko, K.F. Karlsson, P.O. Holtz, B. Monemar, W.V. Schoenfeld, J.M. Garcia, P.M. Petroff. Phys. Rev. B 64, 085302 (2001).

[11] E.S. Moskalenko, K.F. Karlsson, P.O. Holtz, B. Monemar, W.V. Schoenfeld, J.M. Garcia, P.M. Petroff. Phys. Rev. B 66, 19, 195332 (2002).

[12] K.F. Karlsson, E.S. Moskalenko, P.O. Holtz, B. Monemar, W.V. Schoenfeld, J.M. Garcia, P.M. Petroff. Appl Phys. Lett. 78, 19, 2952 (2001).

[13] A.M. Wite, P.J. Dean, D.J. Ashen, G.B. Mullin, B. Webb, B. Day, P.D. Greene. J. Phys. C 6, L243 (1973).

[14] E.J. Johnson, J. Kafalas, R.W. Davis, W.A. Dyes. Appl. Phys. Lett. 40, 11, 993 (1982).

[15] S. Adachi. J. Appl. Phys. 66, 12, 6030 (1989).

[16] P. Blood, J.J. Harris. J. Appl. Phys. 56, 4, 993 (1984).

[17] M. Heiblum, E.E. Mendez, L. Osterling. J. Vac. Sci. Technol. B 2, 2, 233 (1984); I.H. Goodridge. Properties of Gallium Arsenide. Inspec, London (1986).

[18] P. Silverberg, P. Omling, L. Samuelson. Appl. Phys. Lett. 52, 20, 1689 (1988).

[19] C. Lobo, R. Leon, S. Marcinkevičius, W. Yang, P. Sercel, X.Z. Liao, J. Zou, D.J.H. Cockayne. Phys. Rev. B 60, 24, 16647 (1999).

[20] K. Mukai, M. Sugawara. In: Self-Assembled InGaAs/GaAs Quantum Dots. / Ed. by M. Sugawara. Semiconductors and Semimetals. Academic Press, San Diego (1999). Vol. 60. P. 183. 